\title{
The Mount Wilson Ca II K Plage Index Time Series
}

\author{
L. Bertello • R.K. Ulrich · J.E. Boyden
}

Received: 19 January 2010 / Accepted: 1 May 2010 / Published online: 19 May 2010

(C) The Author(s) 2010. This article is published with open access at Springerlink.com

\begin{abstract}
It is well established that both total and spectral solar irradiance are modulated by variable magnetic activity on the solar surface. However, there is still disagreement about the contribution of individual solar features for changes in the solar output, in particular over decadal time scales. Ionized Ca II K line spectroheliograms are one of the major resources for these long-term trend studies, mainly because such measurements have been available now for more than 100 years. In this paper we introduce a new $\mathrm{Ca}$ II $\mathrm{K}$ plage and active network index time series derived from the digitization of almost 40000 photographic solar images that were obtained at the 60-foot solar tower, between 1915 and 1985, as a part of the monitoring program of the Mount Wilson Observatory. We describe here the procedure we applied to calibrate the images and the properties of our new defined index, which is strongly correlated to the average fractional area of the visible solar disk occupied by plages and active network. We show that the long-term variation of this index is in an excellent agreement with the 11-year solar-cycle trend determined from the annual international sunspot numbers series. Our time series agrees also very well with similar indicators derived from a different reduction of the same data base and other Ca II K spectroheliograms long-term synoptic programs, such as those at Kodaikanal Observatory (India), and at the National Solar Observatory at Sacramento Peak (USA). Finally, we show that using appropriate proxies it is possible to extend this time series up to date, making this data set one of the longest $\mathrm{Ca}$ II $\mathrm{K}$ index series currently available.
\end{abstract}

Keywords Chromosphere, active · Solar activity, observations, data analysis

\author{
L. Bertello (凶) \\ National Solar Observatory, 950 North Cherry Avenue, Tucson, AZ, USA \\ e-mail: bertello@noao.edu \\ R.K. Ulrich · J.E. Boyden \\ Department of Physics and Astronomy, University of California, Los Angeles 90095, USA \\ R.K. Ulrich \\ e-mail: ulrich@astro.ucla.edu \\ J.E. Boyden \\ e-mail: boyden@astro.ucla.edu
}




\section{Introduction}

The importance of Ca II K spectroheliogram time series for the study of solar magnetism and irradiance variability is well established. Observations of the solar surface reveal magnetic fields with complex hierarchical structures, evolving on a wide range of different spatial and temporal scales. The most prominent aspect of this variability is the solar cycle of activity, with a period of approximately 11 years for the sunspot numbers and a period of about 22 years for the magnetic polarity. Thirty years of satellite measurements of the Sun's energy output have revealed that also the solar irradiance changes over the full range of time scales from minutes to decades (e.g. Fröhlich and Lean, 2004), and this variability is wavelength dependent (Lean, 2001; Solanki and Unruh, 1998). Empirical models have shown that the variability in solar irradiance is indeed modulated by the area variations of the solar surface magnetic features, to a high degree of correlation (Solanki, 2003; Foukal et al., 2004; Foukal and Lean, 1988). Two of these features, plages and chromospheric magnetic network, account for a significant portion of the Sun's total magnetic flux, UV and EUV variability, that play a critical role in determining the conditions in the heliosphere which directly influence the Earth's magnetosphere (see the references in the paper by Foukal et al. (2009) for a discussion on this subject).

Observations near the core of the ionized calcium K line $(393.37 \mathrm{~nm})$ are one of the most effective tools to investigate the morphology and evolution of both plages and chromospheric magnetic network. These measurements have been available since the early years of the twentieth century and, because of their correlation to the solar irradiance, they have been widely used as proxies to reconstruct the history of the solar magnetism and solar irradiance over the last 100 years and beyond (Foukal, 2003; Foukal et al., 2004). The Mount Wilson archive of ionized Ca II K line spectroheliograms provides a fundamental resource for these studies. The intensity calibration of these images, however, is a very difficult task because the disk brightness and level of background vary from one image to another due to contributions from the quiet Sun limb darkening curve of the Ca II K line, the geometrical distortion introduced by the guider errors that alter the image shape, and the vignetting function produced by the misalignment of the optical axis with respect to the center of the grating. For those observations that include the step wedge densitometer strips it is possible to derive a characteristic curve (also known as Hurter-Driffield curve, or H\&D curve) to interpret the transparency of the photographic plate material in terms of an exposure quantity (de Vaucouleurs, 1968). Unfortunately, the step wedge exposures only began 9 October 1961 making this approach unsuitable for the calibration of the entire database. However, digital filters are convenient tools for extracting the properties of the spatial intensity distribution of an image. In this paper we use a median (low-pass) filter to determine the image background of each $\mathrm{Ca}$ II $\mathrm{K}$ observation. A flat-fielded image is then obtained by dividing the image by its background. Two of the parameters that describe the pixel distribution of this normalized image can be used to define a Ca II K plage index and a plage contrast time series. The very good agreement between these two time series and similar products validates the analysis described in this paper (Foukal et al., 2009; Tlatov, Pevtsov, and Singh, 2009). Finally, we show here that it is possible to extend the length of the plage index time series up to date by using its excellent correlation to the Mount Wilson magnetic plage strength index (MPSI), making this Ca II K index times series one of the longest currently available. 


\section{The Mount Wilson Archive}

Since the start of the 20th century, the monitoring program of the Mount Wilson Observatory has made available to the scientific community a huge number of solar images. The glass and acetate negatives are stored and maintained at the Pasadena (California) office of the Observatories of the Carnegie Institution of Washington. This archive contains over 150000 images of the Sun which were acquired over a time span in excess of 100 years. The archive includes broad-band images called White Light Directs, ionized Ca II K line spectroheliograms and Hydrogen Balmer alpha spectroheliograms including both images of the solar disk and images of prominences above the solar limb. In 2003, the solar physics group at UCLA has begun a project to digitize essentially all of the Ca II $\mathrm{K}$ and broad-band direct images out of the archive, with 12 bits of significant precision and up to 3000 by 3000 spatial pixels. This project has now completed the digitization of the Ca II K spectroheliogram series that began in 1915 and ended in 1985. Almost 40000 solar images and step wedge images (available after 1962) have been extracted and identified with original log-book parameters of observation time and scan format. Data from this reduction are accessible from the project archive on-line at http://www.astro.ucla.edu/ ulrich/MW_SPADP. Figure 1 shows the distribution of solar Ca II K spectroheliograms per year investigated by this study.

The analysis of this data set will permit a variety of retrospective analyses of the state of the solar magnetism and provide a temporal baseline of about 100 years for many solar properties. The chromospheric network is clearly visible on a substantial fraction of the archive images from the $\mathrm{Ca}$ II $\mathrm{K}$ spectroheliogram sequence. A previous version of this database was used by Foukal and Milano (2001) to study the quiet network contribution to solar irradiance variations. These variations are important because they can be used to investigate the influence of the solar luminosity change on the Earth's climate (Foukal et al., 2006). More

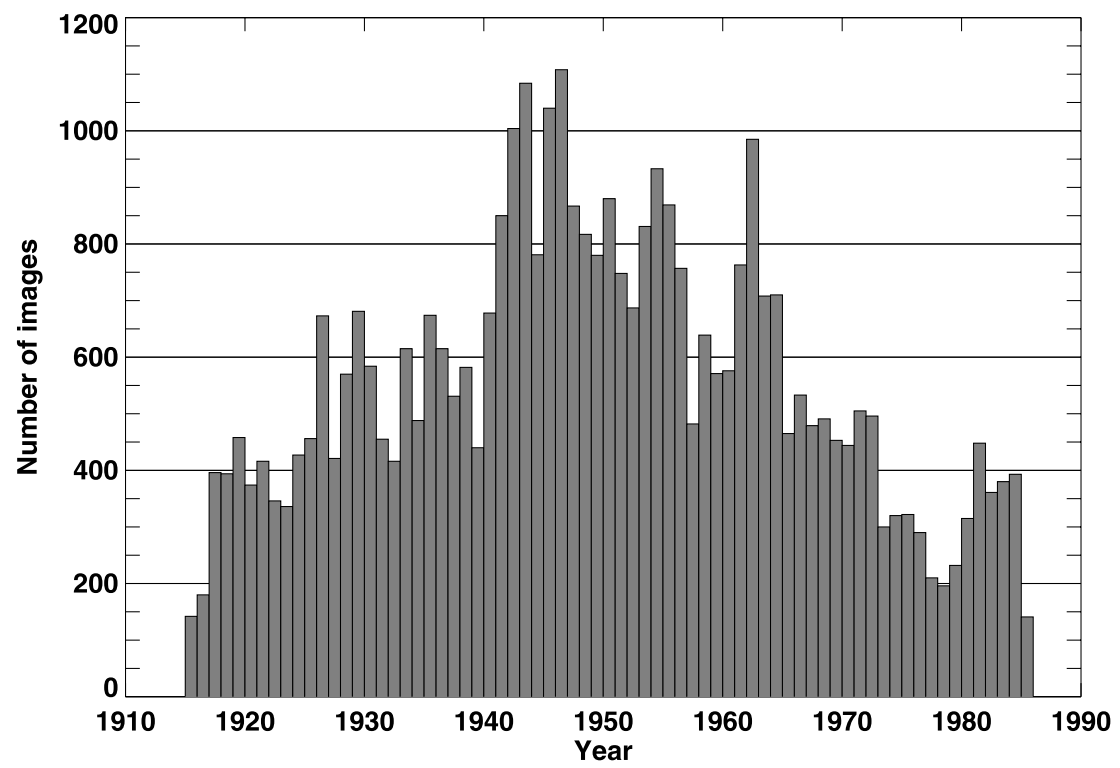

Figure 1 Number of solar Ca II K spectroheliograms per year investigated by this study and also available on line from the project web site. Each image is available as a standard astronomical format fits file that includes information as to date and time of observation as well as key data reduction information (see text). 
recently, the digitized images obtained from this archive were independently calibrated by Tlatov, Pevtsov, and Singh (2009) along with Ca II K data from Kodaikanal Observatory and from the National Solar Observatory at Sacramento Peak, for the purpose of comparing the signature of plages and enhanced network. The comparison shows a good agreement in the temporal behavior of the annual and monthly mean plage indices calculated from the three data sets. A more extended comparison, that includes six time series of annual mean plages indices, shows a similar result (Foukal et al., 2009). The image quality and contents of the $\mathrm{Ca}$ II K spectroheliogram time series obtained by the digitization of the Arcetri, Kodaikanal, and Mt Wilson photographic archives have been also evaluated by Ermolli et al. (2009) to estimate their value for studies focusing on timescales longer than the solar cycle.

\section{Data Analysis}

\subsection{Pre-Processing and Calibration of the Images}

The procedures used to calibrate the Ca II $\mathrm{K}$ images are described in some details at the project web site www.astro.ucla.edu/ ulrich/MW_SPADP. We only provide here a short summary of the most important steps included in this calibration process, relevant to the analysis described in this work. The images present notably some dust and pit which is important to reduce. A Laplacian filter was used for this purpose. The size of the images was then reduced from its original scanned resolution of $\approx 3000 \times 3000$ to $\approx 866 \times 866$ spatial pixels, by averaging the pixel values within each $4 \times 4$ portion of the image. Due to the nature of the scans, where the dispersion direction is in the direction of the scan and the cross-dispersion direction is parallel to the slit, a strategy was adopted whereby the search in the image is for radii associated with the scan/dispersion direction and cross-dispersion/slit direction independently. Because the average distortion of each image amounts to less than $\approx 0.9 \%$, with the radius along the scan/dispersion direction being typically smaller than the radius along the cross-dispersion/slit direction, an average value of the radius was used. Due to small changes in the image-scale size over the years, the annual mean value of this average radius varies from about 336 pixels to 340 pixels, with not significant long-term trends over the 70-year period investigated by this study.

One of the main problems we encountered during the calibration process is the presence of a vignetting function. This function is linked to the relative position between the pupil and the grating, which depends on the coelostat mirror positions and shifts during each exposure due to the scanning of the spectrograph across the solar image. As a result of this effect the intensity and its gradient are highly variable from one image to another. Stepwedge exposures on the Ca II K spectroheliograph sequence are available only since 9 October 1961. For those images it is possible to use, following the ideas presented by de Vaucouleurs (1968), the H\&D curve calibration approach to obtain well calibrated intensity images. Unfortunately, the bulk of the sequence does not offer this possibility and a different approach is required. In our study we used a running median filter to determine the large-scale spatial intensity distribution of the image, i.e. its background. We then divide the image by this background to obtain the final flat-fielded image. Figure 2 illustrates the procedure for a sample of three different observations: The first column shows the original images after they have been corrected for dust and pit and reduced in size. The variation in intensity is clearly visible on all these images. Moreover, the dynamic range is also quite different from one image to another being, for example, much lower in the last image. The second column shows the background determined from the running median filter, and the 


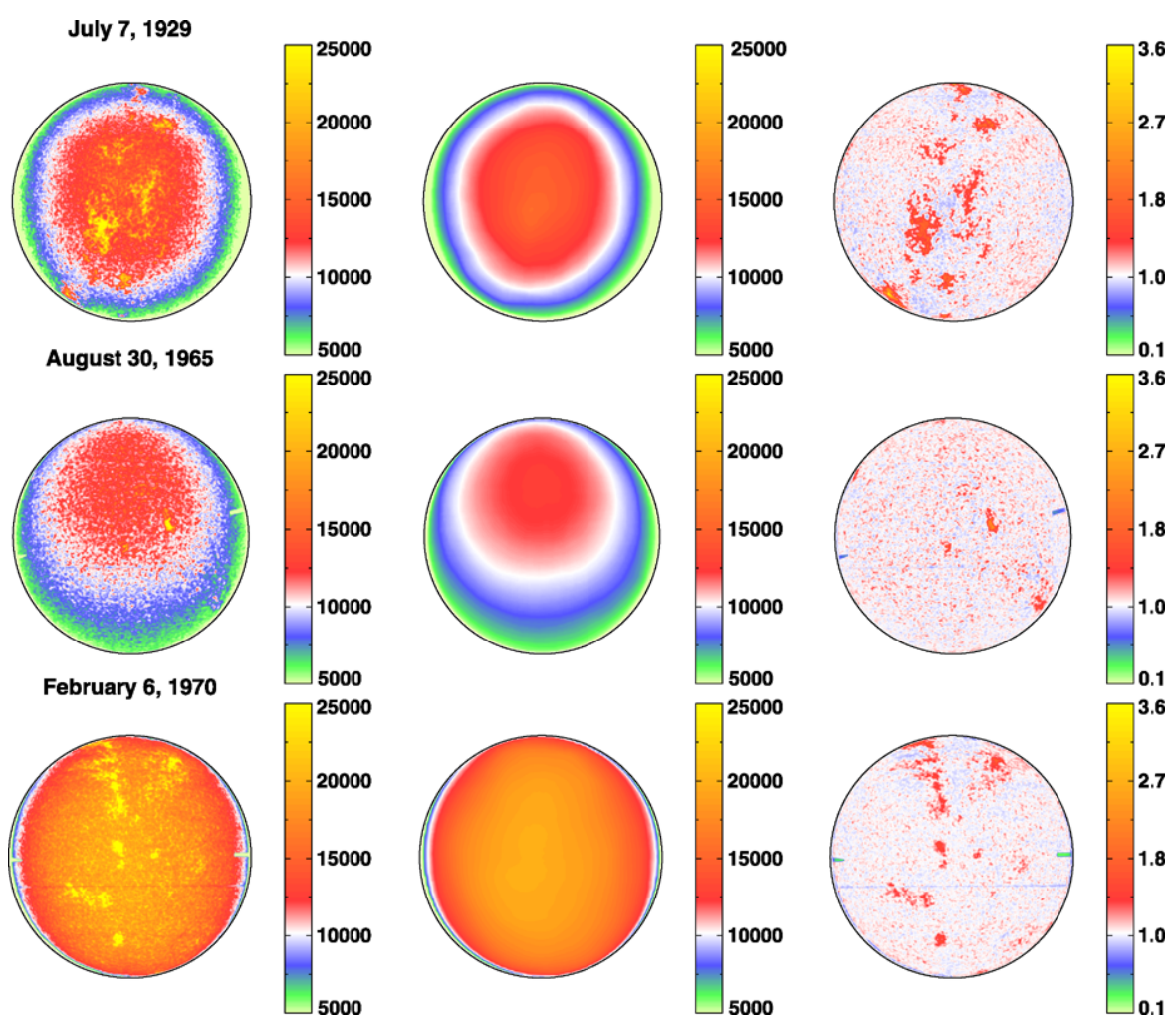

Figure 2 Examples of median-filtered images. The left column shows the original images taken on the days indicated in the figure. The middle column displays the background of the corresponding image calculated using a running median filter, essentially a low-pass filter, and the last column is the result of the flat-fielding obtained by dividing the previous two images together. The chromospheric network is well defined on the final images. Also, on the last two images, the polar marks indicating the solar north-south direction are clearly visible. These marks are present, however, only on a portion of the data set.

last column is the flat-fielded image obtained by dividing the first two images. The parameters of the median filter have been accurately determined by comparing the result of the filter to the proper H\&D calibration for images taken after 9 October 1961. This is an important and necessary step to avoid introducing spurious points through an improper treatment of the non-linear response curve that could affect the values of the $\mathrm{CaK}$ index defined in the next section. The pixel value of the background in the flat-fielded image is typically around unity.

\subsection{The CaK Index Series}

A CaK index is defined from each flat-fielded image using a multi-step procedure based on the distribution of the intensity ratio, as described in details below.

i) A first histogram is calculated using all pixels located within 0.99 solar radii from the center of the image and with values less than three. The bin size of the histogram is chosen to be 0.01 . The width $\sigma$ and the center $x_{\mathrm{c}}\left(x_{\mathrm{c}} \approx 1\right)$ of this distribution are determined from a Gaussian fit to the histogram using an equal number of bins (35) around 
Figure 3 The intensity-ratio distribution for an image taken 2 July 1970. The fundamental parameters of this distribution are determined from a Gaussian fit to the histogram, which contains 30 bins between $x_{\mathrm{c}}-2 \sigma$ and $x_{\mathrm{c}}+7 \sigma$. This is the exact range shown in the plot, where $x_{\mathrm{c}}$ and $\sigma$ are the center and the width of the distribution, respectively. Also shown is the best Gaussian fit to the distribution. The constant baseline $B$ (see Equation (1)) defines the $\mathrm{CaK}$ plages and active network index used in this work.

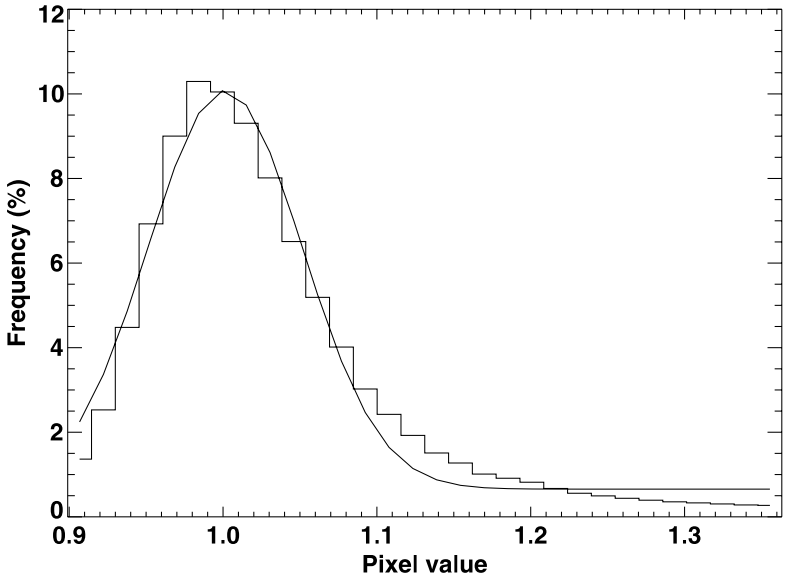

the maximum of the distribution. This choice of parameters limits the analysis to the central part of the histogram, which is well described by a Gaussian function.

ii) The calculated width $\sigma$ is used to define the boundaries of a new histogram, between $x_{\mathrm{c}}-2 \sigma$ and $x_{\mathrm{c}}+7 \sigma$. To properly account for the contribution of both plages and network to the skewed distribution shown in Figure 3, the long tail on the right side of the histogram needs to be included in the fit. We find that a value of $7 \sigma$ for the tail accomplishes this goal and provides results that are in excellent agreement with other studies as shown in this paper. This range is then divided into 30 bins, so that the bin size varies in general from one distribution to another. The histogram is then normalized by dividing the value of each bin by the total number of pixels in the solar disk image. This normalization is necessary, to properly take into account the change in the observed solar disk area produced by the variable Sun - Earth distance.

iii) The following four-parameter Gaussian function is used to model the distribution:

$$
y(x)=A \exp \left(-u^{2} / 2\right)+B
$$

where $u=\left(x-x_{\mathrm{c}}\right) / \sigma, x$ is the bin value, and $y$ is the fractional number of pixels in the solar disk with value $x$.

Figure 3 shows a typical histogram derived from a single observation, defined between the boundaries $x_{\mathrm{c}}-2 \sigma$ and $x_{\mathrm{c}}+7 \sigma$. The solid curve is the Gaussian best fit to the distribution given by Equation (1). It is quite evident from the figure that the distribution is well described by a Gaussian function only for pixel values around to the center $\left(x_{\mathrm{c}} \approx 1\right)$ of the histogram, and tends to drift away from it as the pixel value increases. Since relatively high intensityratio values correspond to brighter than average features in the image, that is plages and enhanced network, the analysis of Figure 3 shows that those features will add contributions to the right side of the histogram and make the distribution asymmetric. The measurement of the constant baseline $B$ from Equation (1) provides an average estimate of this effect.

\subsection{The Ca II K Plages and Active Network Index Series}

The value of the constant baseline $B$ in Equation (1) is defined in this work to be an index of the $\mathrm{Ca}$ II $\mathrm{K}$ plages and active network, or simply a $\mathrm{CaK}$ index. It is important to notice that despite the normalization introduced in this definition, our index does not directly 


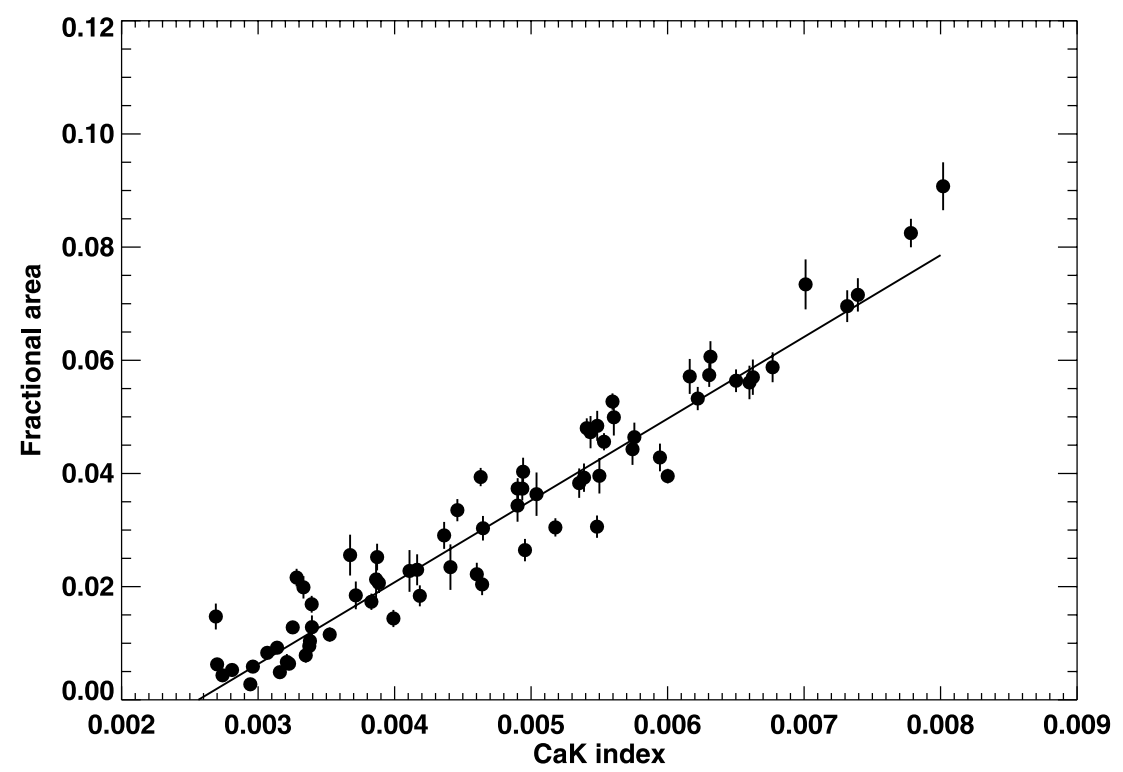

Figure 4 Comparison between the annual means of two indices derived from independent analyses of the Mount Wilson archive of $\mathrm{Ca}$ II $\mathrm{K}$ observations. The $\mathrm{CaK}$ index is the value measured according to the procedure described in this paper, while the fractional areas covered by plages and active network were measured by Tlatov, Pevtsov, and Singh (2009). The annual mean areas were calculated from the monthly values provided by the authors and the error bars are the 1-sigma uncertainties of the mean. For the CaK index, the error bars are much smaller than the size of the points showed in the plot. Also shown is the regression line, given by Equation (2).

measure the fraction of the solar hemisphere occupied by the chromospheric plages and enhanced network. Instead, it measures the average effect of those features on the intensityratio distribution of the image. As discussed in Section 3.1, the lack of information about the characteristic curve for most of the images in the sequence, in addition to the unknown intensity contribution from scattered light, makes the task of obtaining a homogeneous and consistent series of well calibrated images very difficult. Consequently, the calculation of the actual area of the solar disk covered by plages and/or network must rely on some additional assumptions about the physical properties of the database. In their independent analysis of this series, Tlatov, Pevtsov, and Singh (2009) have derived a CaK index expressed in units of fraction of the solar hemisphere covered by plages and network on the assumption that the center-to-limb intensity variation in quiet Sun corresponds to a standard curve, independent of overall level of solar activity. This result can be used to calibrate our time series on the same scale. Figure 4 shows the comparison between our annual means and their corresponding values. The linear Pearson correlation coefficient of these two indices is 0.97 , with a Student t-test showing a significant correlation between the two variables at a confidence level much higher than $99.9 \%$, indicating the excellent agreement of the two series. The linear regression is described by the equation

$$
\text { Fractional area }=-3.70 \times 10^{-2}\left( \pm 0.06 \times 10^{-2}\right)+14.45( \pm 0.15) \mathrm{CaK} \text { index } .
$$

This equation can be used to convert our $\mathrm{CaK}$ index into a fractional area, so that the comparison with similar time series can be made. 


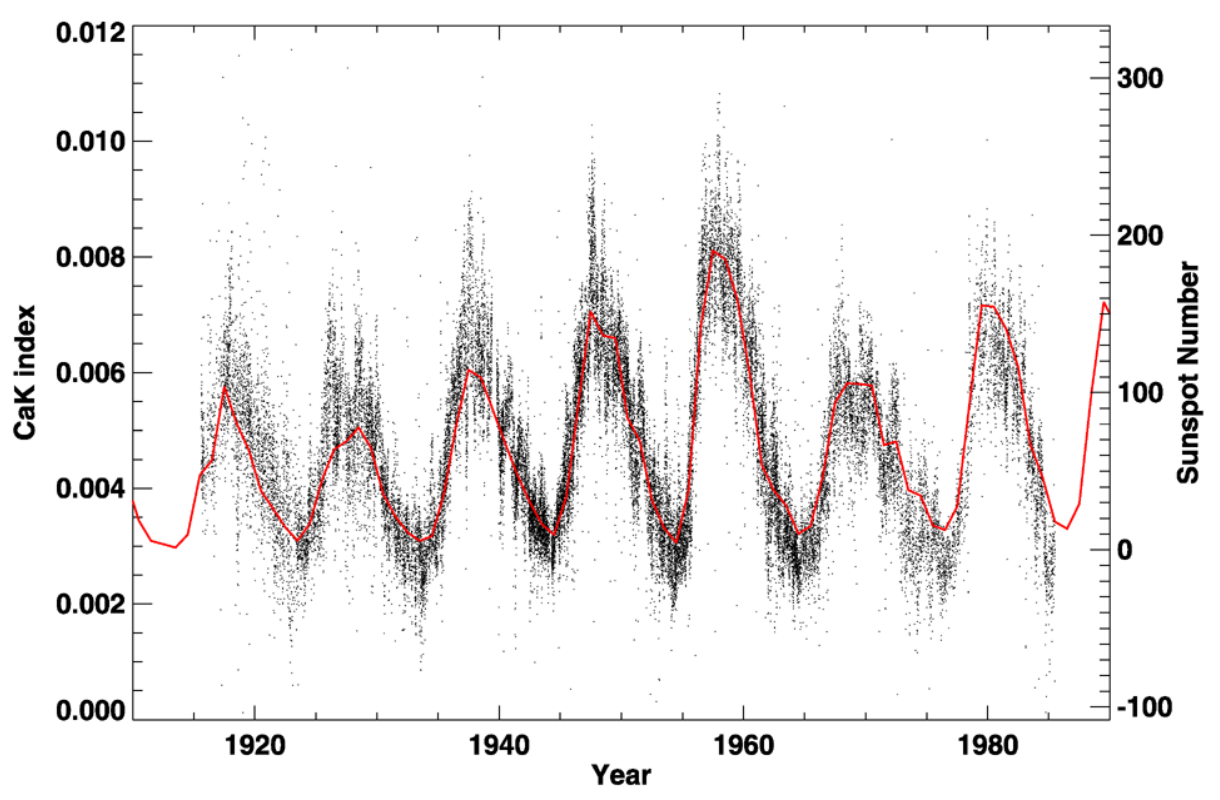

Figure 5 The $\mathrm{CaK}$ index time series defined using the fitting procedure described in the text. This index measures the contribution from both plages and active network. Almost 40000 images have been reduced to produce this plot. The annual international sunspot numbers are also shown, in red, as a reference.

We have applied the procedure described in the previous section to the entire data set of observations taken between 1915 and 1985. The value determined from each image is plotted in Figure 5, together with the temporal annual variations of the international sunspot numbers. No attempt has been done to eliminate any specific observations from this analysis. From the visual inspection of the figure it is quite evident that our $\mathrm{CaK}$ index well correlates with the 11-year solar cycle defined by the sunspot numbers. In addition, comparisons between our $\mathrm{CaK}$ annual mean values and other available $\mathrm{CaK}$ plage indices shows that our definition is consistent with those based on the area of plages including the active network (Foukal et al., 2009; Tlatov, Pevtsov, and Singh, 2009).

In addition to the $\mathrm{CaK}$ index time series, defined by the parameter $B$, the fit to the histogram provides supplementary information about the properties of the distribution in intensity of each image. In particular, the behavior of the width $\sigma$ seems to be correlated to the plage contrast. In their investigation of the Mount Wilson Ca II K photographic archive Tlatov, Pevtsov, and Singh (2009) defined a plage contrast as the plage brightness per unit area. Figure 6 shows the comparison between $\sigma$ and their plage contrast. Although the two quantities have been defined differently and the calibration of the photographic plates was done using completely separated methods, the two curves show very similar variations over time. The plage contrast time series seems to be more sensitive to the variations in the cycle of activity than the result obtained from the histogram analysis. The most interesting feature of this figure is the increasing in the plage contrast during solar cycle 19, from about 1953 to 1961. A similar analysis performed by Tlatov, Pevtsov, and Singh (2009) on the archive of the Kodaikanal Observatory (India) for the period 1907-1999 does not show any significant long-term variation of the plage contrast. It is speculated that the increase in the plage contrast during solar cycle 19 for the Mount Wilson data is due to the exit slit width being narrower during that period (Tlatov, Pevtsov, and Singh, 2009). In fact, an important prop- 


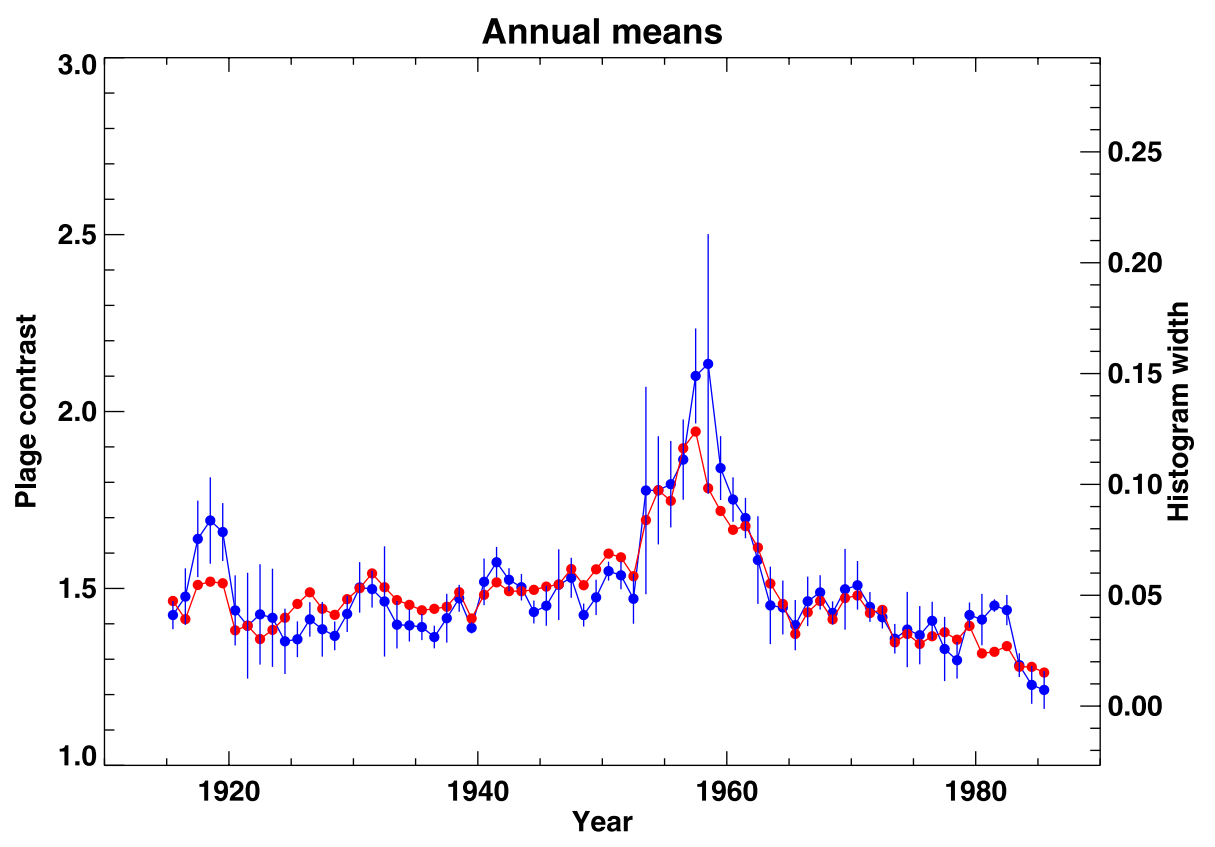

Figure 6 Comparison between the plage contrast (blue) measured by Tlatov, Pevtsov, and Singh (2009) and the annual mean width values (red) derived from the histogram analysis described in this paper. The error bars are three standard deviations of the mean. In our analysis the error bars are much smaller, smaller than the size of the individual (red) points, mainly because of the much large sample used in the investigation.

erty of the images obtained during this interval is the present of dark filaments which are known to be prominences projected onto the solar disk. These features are common for K3 spectroheliograms but not for K2 spectroheliograms, the bulk of this database. The interval for which the filaments are evident coincides closely with this period of anomalous plage contrast.

\subsection{The Extended CaK Index Series}

Since 1970, the synoptic program of Doppler and magnetic observations at the 150-foot solar tower at Mount Wilson has provided to the solar community a MPSI extracted from the Fe I $5250 \AA$ magnetograms. The MPSI is defined as the sum of the absolute values of the magnetic field strengths for all pixels where the absolute value of the magnetic field strength is between 10 and 100 gauss (Chapman and Boyden, 1986). This number is then divided by the total number of pixels (regardless of the magnetic field strength) in the magnetogram. The entire record of MPSI daily measurements is available from www.astro.ucla.edu/ obs. As shown in Figure 7, our daily CaK index is well correlated with the MPSI during the time interval from 1970 to 1985 . In particular, the top plot shows a quite strong linear correlation up to a MPSI value of about two, but then this correlation decreases for larger MPSI numbers. The overall relationship can be well described with a simple three-parameter model, given by $a+b \arctan (c \cdot$ MPSI), where $a, b$, and $c$ are the three parameters to be determined using the method of least squares. Our best fit to the data is shown by the solid red line in top plot of Figure 7. The lower plot shows the temporal behavior of the rescaled MPSI (red line) compared to the corresponding CaK quantity. The MPSI was rescaled using the coefficients 

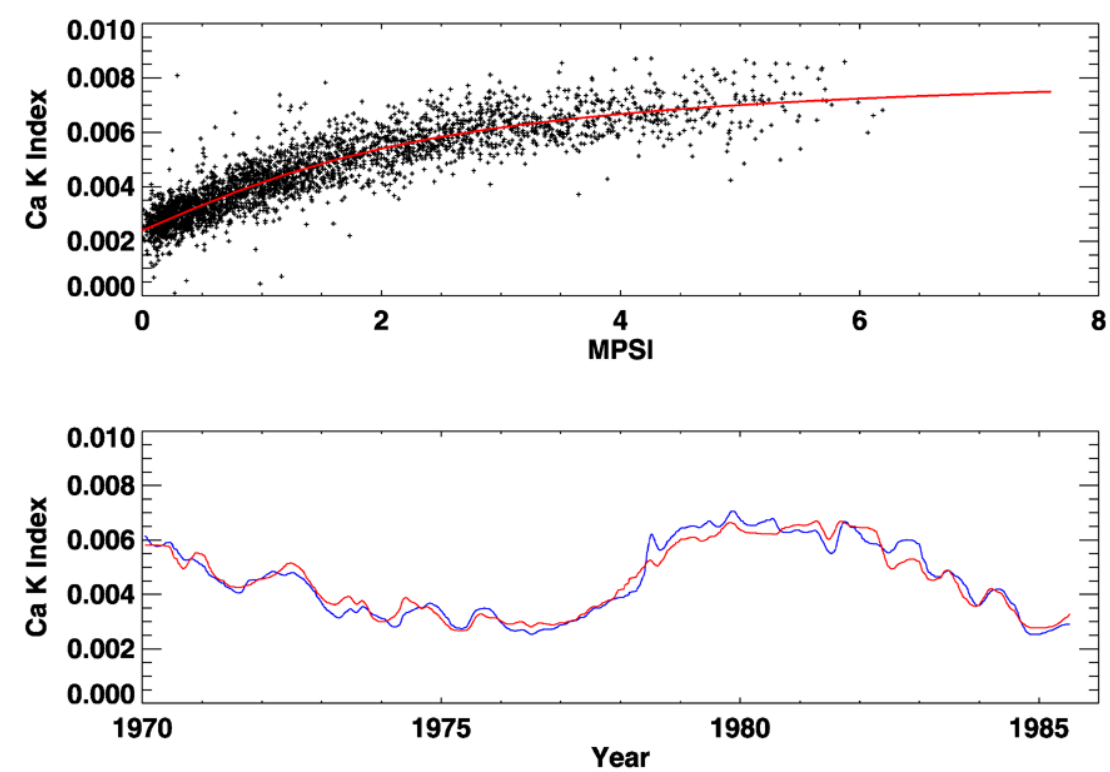

Figure 7 Relationship between the daily CaK index values and MPSI for the time interval 1970-1985. Only days for which both quantities were available have been included in this analysis. This relationship can be described with a simple three-parameter model as described in the text. The solid red line shows the best fit to the data (top plot). A comparison between the temporal variations of the calculated CaK index (blue curve) and the rescaled MPSI (red curve), smoothed using a 60-day width running Gaussian, are also shown (bottom plot).

from the above model. The comparison clearly shows the excellent agreement between the two variables.

An important consequence of this excellent correlation is the possibility of using the MPSI as a proxy for the $\mathrm{CaK}$ index, and therefore extending the calculation of this index up to date. Using the coefficients derived from the model shown in the top plot of Figure 7 we were able to update the series up to 13 July 2009. The annual mean values for the period 1915-2009 are shown in Figure 8, where the blue points are obtained from the 19151985 original spectroheliogram images and the red points from the 1970 -present MPSI measurements. These values are also listed in Table 1 . The most significant feature of this figure, and Table 1, is the fact that the value of our $\mathrm{CaK}$ index during periods of minimum solar activity has slightly but systematically decreased since 1940. In particular, our analysis seems to suggest that the current minimum has produced the lowest $\mathrm{CaK}$ index value among those in the almost hundred years investigated by this study.

\section{Discussion and Conclusions}

In this paper we have described a method to study the time dependence of solar surface magnetism from the analysis of more than 70 years of Ca II K spectroheliograms obtained at the 60-foot solar tower in Mount Wilson between 1915 and 1985. These measurements are important because Ca II $\mathrm{K}$ emission is closely related to the magnetic field (Johannesson, Marquette, and Zirin, 1998) and can provide a proxy for long-term induced changes, solar-cycle and beyond, in the total and spectral solar irradiance (e.g. Fligge, Solanki, and 


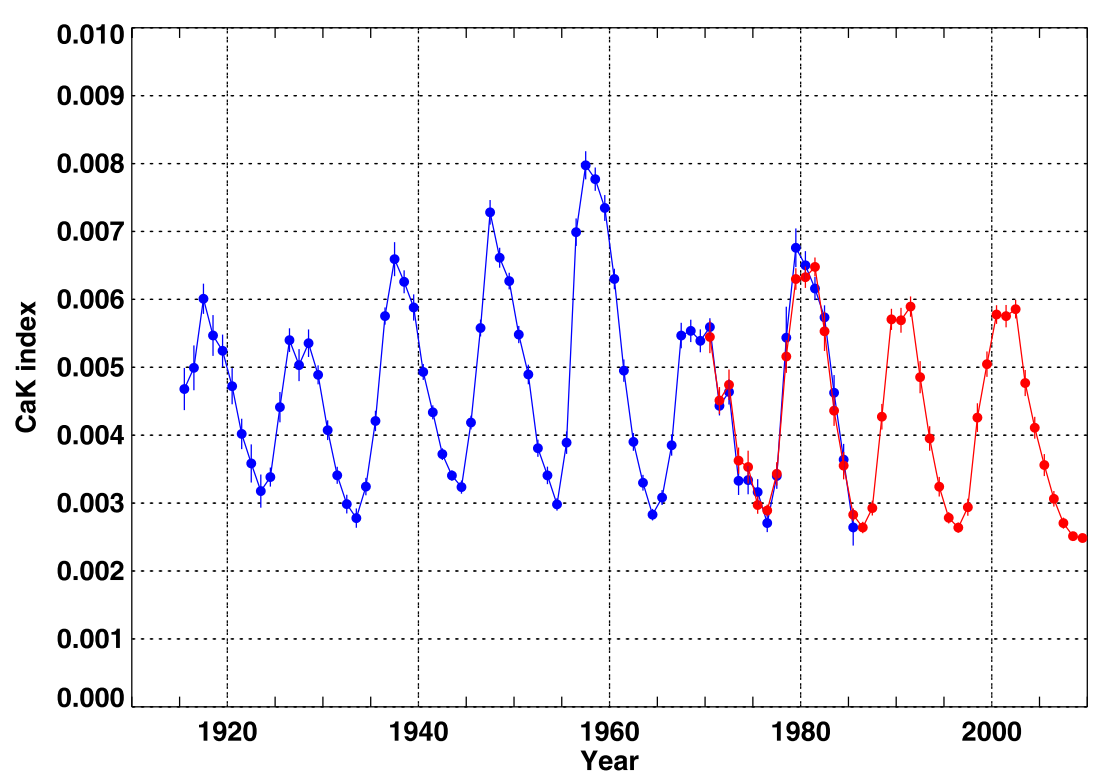

Figure 8 The extended $\mathrm{CaK}$ index series. In blue are the annual mean values obtained from the original spectroheliograms, while in red are the values derived from the MPSI calibrated into the CaK scale. The 16 years overlap between the two series, from 1970 to 1985, show the excellent correlation between the two data sets and validate the use of the MPSI as a proxy to extend the CaK index data. The error bars are 5 times the standard deviation of the mean.

Unruh, 2000). Our method is based on the photometric properties of each individual solar images, using parameters computed from a histogram analysis of their intensity distribution. An important feature of this approach is the fact that it can be accomplished in a fully automated mode, without relying on a visual inspection of the images. We found that two of the parameters defining this intensity distribution, the constant baseline and the width, are very well correlated to the fractional area of the solar disk covered by plages plus active network and the plage contrast as computed by an independent investigation of the same database. The constant baseline measures the strength of the widely distributed regions that are brighter than average in $\mathrm{Ca}$ II $\mathrm{K}$, and it is defined in this work as a CaK index. This index shows a temporal behavior which is in excellent agreement with the cycle of solar activity described by the international sunspot number. This is clearly illustrated in Figure 5, where the relative strength of solar cycles 15 to 21 is well reproduced.

Another remarkable property of this index is its correlation with the MPSI measurements shown in Figure 7. The relationship between these two variables is mostly linear, up to a MPSI value of about two. Above this value the correlation drops quite significantly. Those are measurements corresponding to the time interval from 1979 to 1982.4, around the maximum of solar cycle 21, where both the MPSI and the CaK index show no systematic trends. As illustrated at the bottom of Figure 7, our adopted model produces an excellent agreement between these two quantities during both the period of minimum of activity, around 1975 , and the maximum of cycle 21 . The validity of this model, however, is limited to MPSI values up to about seven. In fact, above this limit, the asymptotic behavior of the model significantly reduces the correlation between these two indices of solar activity. Fortunately, an investigation of the historical record of MPSI daily measurements shows that this event is extremely rare, and will not affect the conclusions reached in this paper. 
Table 1 The annual averages of the CaK time series and the corresponding standard deviation (rms) of the mean. The values after 1985 have been derived from the MPSI time series, used as a proxy (see text).

\begin{tabular}{|c|c|c|c|c|c|c|c|c|}
\hline Year & $\begin{array}{l}\text { Index } \\
\times 10^{-5}\end{array}$ & $\begin{array}{l}\mathrm{rms} \\
\times 10^{-5}\end{array}$ & Year & $\begin{array}{l}\text { Index } \\
\times 10^{-5}\end{array}$ & $\begin{array}{l}\mathrm{rms} \\
\times 10^{-5}\end{array}$ & Year & $\begin{array}{l}\text { Index } \\
\times 10^{-5}\end{array}$ & $\begin{array}{l}\mathrm{rms} \\
\times 10^{-5}\end{array}$ \\
\hline 1915 & 467.98 & 6.22 & 1947 & 728.16 & 3.62 & 1979 & 675.98 & 5.69 \\
\hline 1916 & 499.23 & 6.60 & 1948 & 661.32 & 2.94 & 1980 & 650.16 & 4.18 \\
\hline 1917 & 600.91 & 4.44 & 1949 & 626.94 & 2.46 & 1981 & 615.96 & 3.39 \\
\hline 1918 & 546.83 & 6.01 & 1950 & 548.21 & 2.54 & 1982 & 573.47 & 3.55 \\
\hline 1919 & 524.30 & 4.78 & 1951 & 489.41 & 2.90 & 1983 & 462.49 & 5.21 \\
\hline 1920 & 472.13 & 5.33 & 1952 & 380.80 & 2.53 & 1984 & 364.02 & 4.65 \\
\hline 1921 & 401.88 & 4.47 & 1953 & 340.81 & 2.58 & 1985 & 272.24 & 3.08 \\
\hline 1922 & 358.41 & 5.62 & 1954 & 298.11 & 1.84 & 1986 & 264.05 & 1.68 \\
\hline 1923 & 317.68 & 4.89 & 1955 & 389.04 & 3.31 & 1987 & 292.68 & 2.19 \\
\hline 1924 & 338.34 & 2.80 & 1956 & 698.96 & 4.05 & 1988 & 427.24 & 3.76 \\
\hline 1925 & 441.29 & 4.46 & 1957 & 797.44 & 4.15 & 1989 & 570.57 & 3.10 \\
\hline 1926 & 539.94 & 3.50 & 1958 & 777.01 & 3.43 & 1990 & 569.22 & 3.66 \\
\hline 1927 & 503.16 & 4.71 & 1959 & 734.62 & 3.82 & 1991 & 589.40 & 3.00 \\
\hline 1928 & 535.43 & 4.05 & 1960 & 630.05 & 3.03 & 1992 & 485.43 & 4.73 \\
\hline 1929 & 488.86 & 2.79 & 1961 & 495.05 & 3.29 & 1993 & 395.17 & 3.60 \\
\hline 1930 & 407.35 & 2.90 & 1962 & 390.19 & 2.63 & 1994 & 324.17 & 2.89 \\
\hline 1931 & 340.92 & 2.52 & 1963 & 329.97 & 2.33 & 1995 & 278.37 & 1.67 \\
\hline 1932 & 298.51 & 2.76 & 1964 & 282.97 & 1.70 & 1996 & 263.90 & 1.50 \\
\hline 1933 & 277.89 & 2.85 & 1965 & 308.19 & 2.19 & 1997 & 293.92 & 2.52 \\
\hline 1934 & 324.32 & 2.57 & 1966 & 385.17 & 3.07 & 1998 & 425.89 & 4.23 \\
\hline 1935 & 420.96 & 3.00 & 1967 & 546.75 & 3.77 & 1999 & 504.58 & 3.74 \\
\hline 1936 & 575.43 & 2.53 & 1968 & 553.61 & 3.30 & 2000 & 577.69 & 2.74 \\
\hline 1937 & 659.24 & 5.00 & 1969 & 538.95 & 3.38 & 2001 & 575.31 & 3.30 \\
\hline 1938 & 625.86 & 3.38 & 1970 & 559.24 & 2.59 & 2002 & 585.56 & 2.75 \\
\hline 1939 & 588.09 & 3.86 & 1971 & 443.25 & 2.84 & 2003 & 476.82 & 3.85 \\
\hline 1940 & 493.13 & 2.35 & 1972 & 463.73 & 3.72 & 2004 & 410.99 & 3.23 \\
\hline 1941 & 433.68 & 2.06 & 1973 & 332.84 & 4.18 & 2005 & 356.04 & 3.24 \\
\hline 1942 & 372.09 & 1.72 & 1974 & 333.89 & 4.17 & 2006 & 306.31 & 2.29 \\
\hline 1943 & 340.59 & 1.53 & 1975 & 316.23 & 3.81 & 2007 & 270.50 & 1.63 \\
\hline 1944 & 323.59 & 1.95 & 1976 & 270.64 & 2.67 & 2008 & 251.31 & 1.02 \\
\hline 1945 & 418.65 & 2.03 & 1977 & 339.96 & 3.78 & 2009 & 248.60 & 0.69 \\
\hline 1946 & 557.84 & 2.48 & 1978 & 543.58 & 9.13 & & & \\
\hline
\end{tabular}

The possibility of extending the $\mathrm{CaK}$ index series beyond the time for which the $\mathrm{Ca}$ II $\mathrm{K}$ spectroheliograms are available, using the MPSI measurements as a proxy, is one of the main results of this work. This unique extended series, that covers almost a century of measurements, is shown in Figure 8 and tabulated in Table 1. Because of the close connection among Ca II K emission, solar surface magnetic field, and Total Solar Irradiance (TSI), this CaK time series can provide a proxy for studies on solar-cycle induced changes in the TSI that may play a role in climate change (Solanki and Fligge, 2002; Stott et al., 2000). In particular, the progressive increase in the strength of the solar cy- 


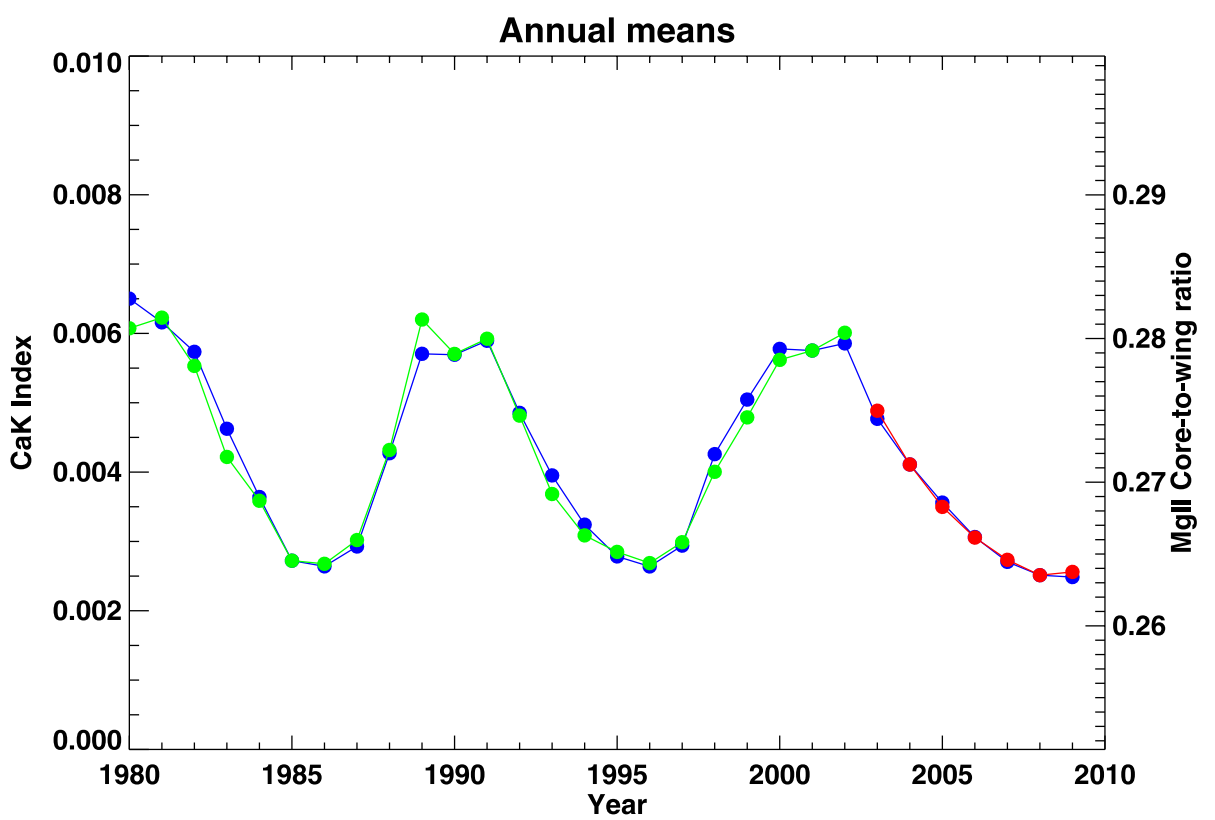

Figure 9 Comparison between the $\mathrm{CaK}$ index (blue points) calculated in this work and the Mg II core-to-wing ratio for the time interval 1980-2009. The Mg II index shown in this figure has been derived using the NOAA Mg II daily index (green points) version 9.1 for the period 1980 to 2002, and the SORCE satellite data (red points) for the period 2003 - present. Both these time series are available from the National Geophysical Data Center website at www.ngdc.noaa.gov/stp/SOLAR/ftpsolaruv.html. Although error bars are not shown in this plot for reason of clarity, the separation between the $\mathrm{CaK}$ index and the corresponding $\mathrm{Mg}$ II points is always less than the cumulative $1-\sigma$ errors.

cle of activity between 1928 and 1958 indicated by the sunspot number is closely tracked by the increase in strength of solar UV radiation as indicated by our $\mathrm{CaK}$ index. This probable increase in the solar output during a period of increasing greenhouse gas abundance needs to be included in evaluations of the global warming process.

Finally, visual inspection of Figure 8 seems to suggest a subtle but systematic decreasing in the value of the $\mathrm{CaK}$ index, around the minima of solar activity, from 1945 to the present. It is well established that chromospheric indices such as $\mathrm{CaK}$ index, $\mathrm{Mg}$ II core-to-wing ratio, and Ly- $\alpha$ are highly correlated with the UV and EUV irradiance. The Mg II index, in particular, has been recently investigated to determine whether the spectral irradiance below about $300 \mathrm{~nm}$ shows some secular changes. Fröhlich (2009) has found evidence of a longterm trend in TSI, but not in solar UV irradiance. The study of the long-term properties of the UV irradiance was, however, limited to the investigation of the temporal behavior of the Mg II index over the last three solar cycles, and confirmed by observations of Ly- $\alpha$ and $\mathrm{Ca}$ II $\mathrm{K}$ over the same time period. In this paper we have the opportunity to significantly extend this time interval, by using our CaK index time series as a proxy for UV and EUV irradiance. This is validated by the high correlation between our $\mathrm{CaK}$ index and the $\mathrm{Mg}$ II core-to-wing ratio shown in Figure 9. Assuming that this correlation is preserved also for the previous cycles, shown in Figure 8, we must conclude that the "quiet" magnetic Sun has slightly and systematically reduced its UV irradiance over the past 70 years. A regression analysis using the $\mathrm{CaK}$ index values from Table 1 . corresponding to years of minimum in solar activity was performed to validate this conclusion. When only the last three or four 
solar minimum values are included in this analysis, the significance (according to a Student t-distribution) of the calculated slope is around or well below the $95 \%$ confidence level, for the four and three minima respectively. This result leads to the conclusion that no significant changes have occurred in the quiet Sun UV irradiance over the last four decades, as also shown by other studies (e.g. Fröhlich, 2009 and references therein). However, when the last six or seven solar minimum values are considered, a significant slope - at a confidence level above $99.9 \%$ - is found. The value of this slope is about 0.9 year $^{-1}$, indicating a variation of $\approx 20-25 \%$ in the value of the "quiet" CaK index between solar cycles 17/18 and $23 / 24$. We are not able to calculate at present how much of this variation, if any, would produce changes in the quiet-Sun's output of UV. If such a variation can be demonstrated, it could significantly impact the study of the possible effect of the Sun on the Earth's climate, as discussed in Foukal et al. (2006). We have repeated this analysis using the smoothed temporal behavior of the daily $\mathrm{CaK}$ index instead of the annual means, and reached the same conclusions. Further investigation and comparison with other data sets are required, however, to positively confirm this result.

Acknowledgement This work has been supported by NSF grant to UCLA NSF ATM-0236682.

Open Access This article is distributed under the terms of the Creative Commons Attribution Noncommercial License which permits any noncommercial use, distribution, and reproduction in any medium, provided the original author(s) and source are credited.

\section{References}

Chapman, G.A., Boyden, J.E.: 1986, Astrophys. J. Lett. 302, 71.

de Vaucouleurs, G.: 1968, Appl. Opt. 7, 1513.

Ermolli, I., Solanki, S.K., Tlatov, A.G., Krivova, N.A., Ulrich, R.K., Singh, J.: 2009, Astrophys. J. 698, 1000.

Fligge, M., Solanki, S.K., Unruh, Y.C.: 2000, Space Sci. Rev. 94, 139.

Foukal, P.: 2003, EOS Trans. 84, 205.

Foukal, P., Lean, J.: 1988, Astrophys. J. 328, 347.

Foukal, P., Milano, L.: 2001, Geophys. Res. Lett. 28, 883.

Foukal, P., Bernasconi, P., Eaton, H., Rust, D.: 2004, Astrophys. J. Lett. 611, 57.

Foukal, P., Fröhlich, C., Spruit, H., Wigley, T.M.L.: 2006, Nature 443, 161.

Foukal, P., Bertello, L., Livingston, W.C., Pevtsov, A.A., Singh, J., Tlatov, A.G., Ulrich, R.K.: 2009, Solar Phys. 255, 229.

Fröhlich, C.: 2009, Astron. Astrophys. 501, L27.

Fröhlich, C., Lean, J.: 2004, Astron. Astrophys. Rev. 12, 273.

Johannesson, A., Marquette, W.H., Zirin, H.: 1998, Solar Phys. 177, 265.

Lean, J.L.: 2001, Geophys. Res. Lett. 28, 4119.

Solanki, S.K.: 2003, AGU, Fall Meeting, SH31C-01.

Solanki, S.K., Fligge, M.: 2002, Adv. Space Res. 29, 1933.

Solanki, S.K., Unruh, Y.C.: 1998, Astron. Astrophys. 329, 747.

Stott, P.A., Tett, S.F.B., Jones, G.S., Allen, M.R., Ingram, W.J., Mitchell, J.F.B.: 2000, Space Sci. Rev. 94, 337.

Tlatov, A.G., Pevtsov, A.A., Singh, J.: 2009, Solar Phys. 255, 239. 